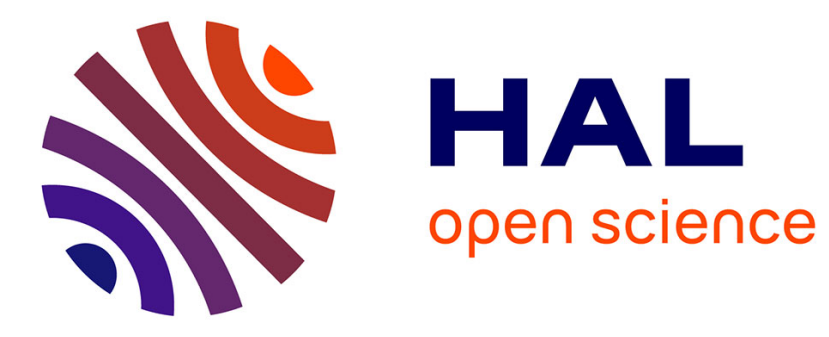

\title{
Kriging for eddy-current testing problems
}

Sandor Bilicz, Emmanuel Vazquez, Szabolcs Gyimothy, Jozsef Pavo, Marc

Lambert

\section{To cite this version:}

Sandor Bilicz, Emmanuel Vazquez, Szabolcs Gyimothy, Jozsef Pavo, Marc Lambert. Kriging for eddy-current testing problems. 17th Conference on the Computation of Electromagnetic Fields (COMPUMAG'09), Nov 2009, Florianopolis, Brazil. pp.3-4. hal-00438557v2

\section{HAL Id: hal-00438557 \\ https://hal.science/hal-00438557v2}

Submitted on 4 Dec 2009

HAL is a multi-disciplinary open access archive for the deposit and dissemination of scientific research documents, whether they are published or not. The documents may come from teaching and research institutions in France or abroad, or from public or private research centers.
L'archive ouverte pluridisciplinaire HAL, est destinée au dépôt et à la diffusion de documents scientifiques de niveau recherche, publiés ou non, émanant des établissements d'enseignement et de recherche français ou étrangers, des laboratoires publics ou privés. 


\title{
Kriging for eddy-current testing problems
}

\author{
S. Bilicz ${ }^{* \dagger}$, E. Vazquez ${ }^{\ddagger}$, Sz. Gyimóthy ${ }^{\dagger}, J^{\prime}$ Pávó ${ }^{\dagger}$, M. Lambert * \\ * Département de Recherche en Électromagnétisme, Laboratoire des Signaux et Systèmes UMR8506 \\ (CNRS-SUPELEC-Univ Paris-Sud), 91192 Gif-sur-Yvette cedex, France \\ $\dagger$ Budapest University of Technology and Economics, Egry J. u. 18, H-1521 Budapest, Hungary, \\ † SUPELEC, 91192 Gif-sur-Yvette cedex, France \\ E-mail: bilicz@evt.bme.hu
}

\begin{abstract}
Accurate numerical simulation of eddy-current testing (ECT) experiments usually requires large computational efforts. To avoid time-consuming computations, a natural idea is to build a cheap approximation of the expensive-torun simulator. In this paper, a kriging-based approximation of an ECT simulator is presented. Kriging is widely used in other domains, but is still quite unexplored in the ECT community. The kriging approximation is built using a random process model of the simulator and a set of simulation results obtained for a number of different input configurations. The resulting approximation might yield almost the same results as those of the simulator.
\end{abstract}

Keywords: Eddy-current testing; Kriging; simulator approximation

\section{INTRODUCTION}

Eddy-current testing is well-known for a long time and is applied in a wide range of industrial problems. However, the accurate numerical simulation of the physical phenomenon is still challenging. Finite element and/or integral equation methods are able to provide acceptable accuracy, at a quite high price however, since these methods are implemented using technically complex computer programs that generally necessitate advanced hardware and long computation times. Nowadays, more and more emphasis is put on the emulation, or surrogate modeling of EM phenomena [1]. This trend leads the electromagnetic community to consider new methods based on mathematical tools from other domains.

Our paper presents an application of kriging to eddycurrent testing problems. The proposed method provides a way to construct a cheap approximation for a specific ECT problem. The main idea is to build a database of ECT configurations, i.e. a set of pairs consisting of a vector of the parameters of a defect and the corresponding output signal, and then to use kriging to predict the output signal at any untried vector of defect parameters. Once the database is built, one does not need the expensive simulator any longer, but only a cheap kriging model.

Kriging is a prediction method appeared in the $60 \mathrm{~s}$ in the domain of geostatistic. By now, several variants of the method have been developed and a comprehensive literature deals with kriging (see, e.g. [2]). For a recent review of the topic, see [3]. Some applications in electromagnetics are presented for instance in [4]. Also the authors have applied kriging to solve ECT inverse problems in [5]. However, the approach of [5] focuses on the kriging prediction of a cost-function (to be minimized in an optimization loop) - now the present paper deals with the use of kriging directly on the output signal.

\section{THE PROPOSED METHOD}

\section{A. The forward prediction problem}

The cheap input-output approximation will be built from a database of computer simulations of the ECT problem. To simplify the notation, let us suppose that a vector of output parameters $\mathbf{y}$ is related to a vector of input parameters $\mathbf{x}$ through

$$
\mathbf{y}=\mathbf{f}(\mathbf{x}),
$$

f representing the underlying physical phenomena. For eddy-current testing application $\mathbf{x}$ might be the geometrical parameters describing a defect affecting a plate and $\mathbf{y}$ the measured data (coil impedances at different locations above the plate). A possible realization $\mathbf{f}$ is a numerical solution of the corresponding Maxwell's equations.

The proposed method is a two-step approach.

1) Compute some corresponding $\left(\mathbf{x}_{i}, \mathbf{y}_{i}\right), i=1, \ldots, n$ pairs ("samples") using (1) and store them in a database that one will use at the stage 2.

2) Fit a kriging interpolator to the samples stored in the database and predict the sought $\mathbf{y}$ output at any arbitrary $\mathbf{x}$ input point.

\section{B. Basics of kriging}

The main idea of kriging is to model a real-valued function $f$ by a Gaussian process, the latter being characterized by its mean and its covariance function. The covariance function describes the statistical dependence between two values of the process and it is a scalar function of one parameter under the hypothesis of a stationary modeling process. However, the covariance function is not known before $f$ has been observed. Generally, one chooses a class of parameterized covariance functions and tunes the parameters in order to fit the modeling process to the pointwise observations of $f$.

Once an appropriate covariance function is determined, the prediction $\hat{f}(x)$ of $f(x)$ at any arbitrary $x$ is written as a linear combination of the observations:

$$
\hat{f}(x)=\sum_{i=1}^{n} \lambda_{i}(x) f\left(x_{i}\right) .
$$

The $\lambda_{i}(x)$ weights are computed using the covariance function, by solving a linear system of $n$ equations. The prediction is an interpolation, since $f\left(x_{k}\right)=\hat{f}\left(x_{k}\right)$ holds for all $k=1,2, \ldots, n$. Note that beyond the mere interpolation, kriging provides additional information about the uncertainty of the prediction as well.

\section{Application to the ECT problem}

In (1), the output $\mathbf{y}$ is a vector of size $q$. It is then appropriate to use an extension of kriging called co-kriging 
[2] to obtain a prediction model, which takes into account, not only the statistical dependence between the inputs and each component of the vector-valued output, but also the dependence between the components of the output vector. Formally, the kriging prediction is

$$
\hat{\mathbf{y}}(\mathbf{x})=\sum_{i=1}^{n} \boldsymbol{\Lambda}_{i}(\mathbf{x}) \mathbf{y}_{i}
$$

with the weighting matrices $\boldsymbol{\Lambda}_{i}(\mathbf{x})$ of size $q \times q$. The use of $n$ pre-computed samples $\left(\mathbf{y}_{i}=\mathbf{y}\left(\mathbf{x}_{\mathbf{i}}\right), i=1,2, \ldots, n\right)$ leads to a linear system of $n \times q$ equations to solve which can be computationally demanding. The hypothesis that all entries of $\mathbf{y}$ have similar statistical behaviour leads to a simpler problem of $q$ distinct kriging models (one for each entry of $\mathbf{y}$ ). Due to the assumed statistical similarity of entries, the models are the same and one can then write

$$
\hat{\mathbf{y}}^{*}(\mathbf{x})=\sum_{i=1}^{n} \lambda_{i}(\mathbf{x}) \mathbf{y}_{i}
$$

using the weighting scalar $\lambda_{i}(\mathbf{x})$ obtained through the solution of a system of only $n$ linear equations. The common covariance (describing the behaviour of all entries of $\mathbf{y}$ ) can be either chosen "by hand" or fitted by maximum likelihood to an appropriate one-dimensional representation of $\mathbf{y}$ (e.g. the first principal component).

The (3) expression is a convenient and computationally cheap interpolator. Beyond the introduction of the method, the main aim of our paper is to examine the validity of the assumptions (leading from the exact (2) to the treatable (3) predictions).

\section{Illustrative ECT PROBlem}

In the studied ECT configuration, a volumetric defect (with a $\sigma(\mathbf{r})$ variable conductivity) takes place within a homogeneous, non-magnetic conductive plate (with $\sigma_{0}$ conductivity). An air-cored pancake type coil (probe) scans above the damaged zone, in a plane parallel to the plate. The coil is driven by sinusoidal current and the variations of its impedance at different locations are measured. These locations are placed at the nodes of a rectangular grid, i.e. a surface scan is performed. The defect is assumed to be cuboid-shaped of volume $\Omega$, thus, can be described by a small number of geometrical parameters, such as sizes and positions. The solution of the forward problem is obtained by the classical volume integral approach [6]. The electric field at a position $\mathbf{r}$ in the plate is written as a sum of two terms: $\mathbf{E}(\mathbf{r})=\mathbf{E}^{i}(\mathbf{r})+\mathbf{E}^{d}(\mathbf{r})$ where $\mathbf{E}^{i}$ is the incident field (in a flawless plate), and $\mathbf{E}^{d}$ is the defect field, i.e. the distortion of the field due to the flaw. The current dipole densities $\mathbf{P}$ and $\mathbf{P}^{\mathbf{i}}$ are defined by $\mathbf{P}(\mathbf{r})=\left(\sigma(\mathbf{r})-\sigma_{0}\right) \mathbf{E}(\mathbf{r})$ and $\mathbf{P}^{i}(\mathbf{r})=\left(\sigma(\mathbf{r})-\sigma_{0}\right) \mathbf{E}^{i}(\mathbf{r})$. The interaction of the EM field of the coil with the flaw can be described by an integral equation given by

$$
\mathbf{P}(\mathbf{r})=\mathbf{P}^{i}(\mathbf{r})-j \omega \mu_{0} \sigma_{0} \chi(\mathbf{r}) \int_{\Omega} \mathcal{G}\left(\mathbf{r} \mid \mathbf{r}^{\prime}\right) \mathbf{P}\left(\mathbf{r}^{\prime}\right) \mathrm{d} V^{\prime} .
$$

The so-called defect description function $\chi(\mathbf{r})$ is defined as $\chi(\mathbf{r})=\left[\sigma(\mathbf{r})-\sigma_{0}\right] / \sigma_{0} \cdot \mathcal{G}\left(\mathbf{r} \mid \mathbf{r}^{\prime}\right)$ is nothing but the classical dyadic Green's function. Once (4) is solved, the variation of the coil impedance can be expressed as

$$
\Delta Z=-\frac{1}{I_{0}^{2}} \int_{\Omega} \mathbf{E}^{i}(\mathbf{r}) \cdot \mathbf{P}(\mathbf{r}) \mathrm{d} V,
$$

based on the reciprocity theorem. Here, $I_{0}$ denotes the current of the probe coil.

A simple case with two input parameters is chosen to illustrate the method. An OD-type defect (having $\sigma(\mathbf{r})=0$ everywhere in $\Omega$ ) with a known position and width takes place within the plate. The defect is characterized by its length $(L)$ and depth ( $D$, given in \% of plate thickness) leading to a 2-dimensional input parameter vector $\mathbf{x}$. The surface scan of the probe coil is centered on the crack and contains $q=841$ measurement points and the corresponding $q \Delta Z$ complex values (5) are stored in the output parameter vector $\mathbf{y}$.

The region of interest in the input domain is $0.5 \mathrm{~mm}<$ $L<3.5 \mathrm{~mm}$ for the lenght and $10 \%<D<90 \%$ for the depth, respectively. In Fig. 1, one can see the input samples $(5 \times 5$ regularly spaced $)$ and the normalized error of the prediction by (3).

The result is promisingly nice: a very small $(\approx 2 \%)$ prediction error, by using only $n=25$ samples (which means that the kriging prediction is made in no time).

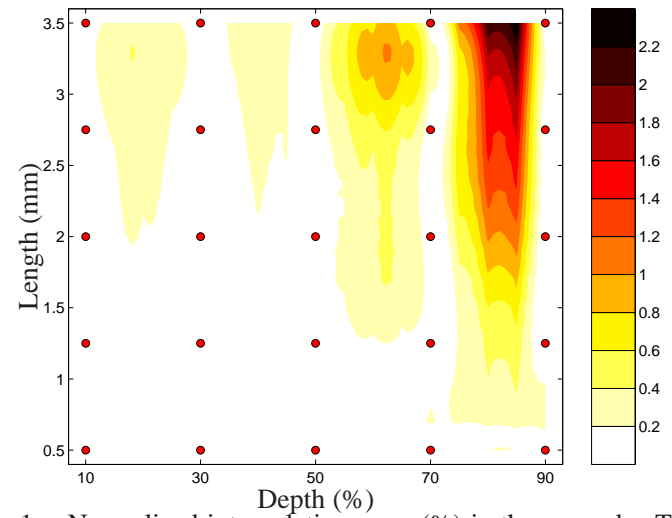

Figure 1. Normalized interpolation error $(\%)$ in the example. The normalizing term is $\|\mathbf{y}\|_{2}$ of the largest $(L=3.5 \mathrm{~mm}, D=90 \%)$ defect.

\section{Conclusion AND Future work}

A kriging-based approach as a cheap approximation for ECT problems is presented. In the light of the preliminary results, the method is promising. However, a precise examination of the validity of the simplifications is the main task for the future.

Though no theoretical limitation restricts the method to the domain of ECT problems, we described and formalized our approach in the context of a given ECT configuration. However, generality is not narrowed in this way, one can also easily imagine other applications.

\section{REFERENCES}

[1] J. K. Sykulski, "New trends in optimization in electromagnetics," Przeglad Elektrotechniczny, vol. 83, no. 6, 2007.

[2] J. Chiles and P. Delfiner, Geostatistics, Modeling Spatial Uncertainty. Wiley, 1999.

[3] J. P. Kleijnen, "Kriging metamodeling in simulation: A review," European Journal of Operational Research, vol. 192, no. 3, 2009.

[4] L. Lebensztajn, C. A. R. Marretto, M. C. Costa, and J. L. Coulomb, "Kriging: a useful tool for electromagnetic device optimization," IEEE Transaction on Magnetics, vol. 40, no. 2, 2004.

[5] S. Bilicz, E. Vazquez, M. Lambert, S. Gyimóthy, and J. Pávó, "Characterization of a 3D defect using the Expected Improvement algorithm," in Proc. of the 13th IGTE Symposium, Austria, 2008.

[6] J. Bowler and S. Jenkins, "Eddy-current probe impedance due to a volumetric flaw," Journal of Applied Physics, vol. 70, no. 3, 1991. 